\title{
ULTRALUMINOUS X-RAY SOURCE CORRELATIONS WITH STAR-FORMING REGIONS
}

\author{
Douglas A. Swartz ${ }^{1}$ Allyn F. Tennant ${ }^{2}$, And Roberto Soria ${ }^{3}$ \\ Submitted to Astrophysical Journal
}

\begin{abstract}
Maps of low-inclination nearby galaxies in Sloan Digitized Sky Survey $u-g, g-r$ and $r-i$ colors are used to determine whether Ultraluminous X-ray sources (ULXs) are predominantly associated with star-forming regions of their host galaxies. An empirical selection criterion is derived from colors of $\mathrm{H}$ II regions in M 81 and M 101 that differentiates between the young, blue stellar component and the older disk and bulge population. This criterion is applied to a sample of 58 galaxies of Hubble type $\mathrm{S} 0$ and later and verified through an application of Fisher's linear discriminant analysis. It is found that $60 \%(49 \%)$ of ULXs in optically-bright environments are within regions blueward of their host galaxy's H II regions compared to only $27 \%(0 \%)$ of a control sample according to the empirical (Fisher) criterion. This is an excess of $3 \sigma$ above the $32 \%$ (27\%) expected if the ULXs were randomly distributed within their galactic hosts. This indicates a ULX preference for young, $\lesssim 10 \mathrm{Myr}$, OB associations. However, none of the ULX environments have the morphology and optical brightness suggestive of a massive young super star cluster though several are in extended or crowded star-forming (blue) environments that may contain clusters unresolved by Sloan imaging. Ten of the 12 ULX candidates with estimated X-ray luminosities in excess of $3 \times 10^{39} \mathrm{ergs} \mathrm{s}^{-1}$ are equally divided among the group of ULX environments redward of H II regions and the group of optically faint regions. This likely indicates that the brightest ULXs turn on at a time somewhat later than typical of $\mathrm{H}$ II regions; say 10-20 Myr after star formation has ended. This would be consistent with the onset of an accretion phase as the donor star ascends the giant branch if the donor is an $\lesssim 20 \mathrm{M}_{\odot}$ star.
\end{abstract}

Subject headings: galaxies: general - X-rays: galaxies - X-rays: general

\section{INTRODUCTION}

Ultraluminous X-ray sources (ULXs) are defined as the most X-ray luminous off-nucleus point-like objects in nearby galaxies. Their high luminosities $\left(L_{\mathrm{X}} \gtrsim\right.$ $10^{39} \mathrm{ergs} \mathrm{s}^{-1}$ in the few tenths to $\sim 10 \mathrm{keV}$ range) imply high accretor masses; hence their interest as a possible evolutionary link between stellar-mass black holes $\left(M_{\mathrm{BH}} \sim 1-20 \mathrm{M}_{\odot}\right)$ known throughout the Local Group and super-massive black holes $\left(M_{\mathrm{BH}} \sim 10^{6}-10^{9} \mathrm{M}_{\odot}\right)$ ubiquitous in the nuclei of galaxies.

Something beyond normal single-star evolution in the current epoch is required if ULXs are found to have masses much higher than $\sim 100 \mathrm{M}_{\odot}$ (Yungelson et al., 2008; Ohkubo et al., 2006; Heger et al., 2003; Heger \& Woosley, 2002). Compact objects with masses $\gtrsim 100 \mathrm{M}_{\odot}$ are often referred to as intermediate-mass black holes, or IMBHs, to emphasize this non-standard evolutionary path.

Star formation occurs on a wide range of mass scales, even in the strongest starbursts, from the common small OB associations up to the rare massive compact superstar clusters (SSCs). This hierarchy reflects the mass distribution of their originating interstellar clouds and the different conditions present at the time of star formation. For instance, the most massive clusters form only in high-pressure regions such as the high density portions of dwarf galaxies and galactic nuclei or the shocked regions of interacting galaxies (Elmegreen \& Efremov, 1997). Presumably, the most massive stars also form only in the most massive clusters (e.g., Elmegreen, 2004; Weidner \& Kroupa, 2006).

Perhaps a similar hierarchical association between ULXs and their local environments exists with the lowerluminosity, presumably lower-mass, ULXs affiliated with small unbound OB associations and only the extremely luminous, potentially IMBH, objects coincident with SSCs. Certainly, the observed correlation of ULXs with global star formation rate in spirals (Swartz et al., 2004; Gilfanov et al., 2004; Liu et al., 2006) implies that many bright ULXs are associated with the short-lived population of massive stars. ULXs are also more common and more luminous in starburst and interacting/merging galaxies than in normal galaxies and they occur at elevated rates in dwarf galaxies (Swartz et al., 2008). Theoretically, the most promising evolutionary path for IMBHs (see the review by van der Marel, 2004, for other possibilities) is merging of massive stars in the most dense young star clusters (e.g., Portegies Zwart et al., 2004).

What is needed is a systematic study of the local environments of ULXs to quantify any association ULXs may have with particular stellar systems and to quantify the properties (e.g., age and mass) of these systems. In this way additional constraints on the nature of ULXs can be inferred.

Constraints on the age of some ULXs and of their surrounding stellar populations have been obtained from high-spatial-resolution optical studies of individual objects using Hubble or ground-based 8-m class telescopes. These

\footnotetext{
${ }^{1}$ Universities Space Research Association, VP62, NASA Marshall Space Flight Center, Huntsville, AL, USA

${ }^{3}$ Space Science Office, VP62, NASA Marshall Space Flight Center, Huntsville, AL, USA

${ }^{3}$ Mullard Space Science Laboratory, University College London, Holmbury St. Mary, Surrey RH5 6NT, UK
} 
studies have found several examples of late O or early B stars as either likely mass donors (Liu et al., 2002, 2004; Kuntz et al., 2005; Ptak et al., 2006; Terashima et al., 2006; Roberts et al., 2008) or populating the nearby environment (e.g., Soria et al., 2005; Grisé et al., 2008). In some cases, emission nebulae have been found associated with ULXs (Pakull \& Mirioni, 2002; Pakull et al., 2006). These results suggest the more common ULX environment is a small $\mathrm{OB}$ association rather than the compact core of a massive SSC. That is, if the hierarchical argument has any merit, the observational results to date argue against an IMBH association for ULXs.

However, these studies are feasible only for a few nearby ULXs and are limited to those ULXs with luminous donors or at least to those located in OB associations. Hence, they may not be representative of the average ULX population. In fact, there are ULXs in elliptical galaxies. These likely have low-mass donors fainter than current detection limits (e.g., Ptak et al., 2006; Roberts et al., 2008).

Here, a different approach is used to quantify the strength of the association between individual ULXs and local stellar populations that is applicable to larger samples of galaxies and out to larger distances where confusion prevents studies of individual stars around a ULX. Specifically, our goal is to determine what fraction of ULXs are associated with HII regions (currently star-forming regions, or with an age $\lesssim 10 \mathrm{Myr}$ since the end of star formation), and what fraction is spatially coincident with young, massive star clusters. To do so, we rely on areaaveraged optical colors, instead of individual bright stars, using the uniform, high-precision, multi-band photometry provided by the Sloan Digitized Sky Survey (SDSS). In particular, the SDSS $u$ band lies blueward of the $D(4000)$ break (Bruzual, 1983) known to distinguish young from old stellar populations in SDSS data (e.g., Kauffmann et al., 2003) while $u-r$ or $u-g$ colors can distinguish early- from late-type galaxies in general (Strateva, 2001; Baldry et al., 2004).

A description of the survey sample, details of the reduction of SDSS images to color maps, our definition of local environment, and a summary of the X-ray data used in this work are presented in $\S 2$. An empirical criterion to distinguish star-forming regions from non-star-forming regions using SDSS colors is developed in $\S 3$ along with a complementary analysis using Fisher's linear discriminant analysis (Fisher, 1936). Results of applying these criteria are given in $\S 4$ and the implications for ULXs is discussed in $\S 5$.

\section{SAMPLE SELECTION}

The galaxies included in this study are a subset of a complete sample of 140 galaxies selected for X-ray study of their ULX populations. The X-ray sample was chosen to form a volume-limited set of galaxies that are in both the set of all galaxies within $15 \mathrm{Mpc}$ contained in the Uppsala Galaxy Catalog with photographic magnitude $m_{p}<14.5 \mathrm{mag}$ and the set of all galaxies listed in the Infrared Astronomical Satellite (IRAS) catalogs with a flux $f_{\mathrm{FIR}} \geq 10^{-13.3} \mathrm{ergs} \mathrm{cm}^{-2} \mathrm{~s}^{-1}$. The UGC (Nilson, 1973) contains all galaxies north of B1950 $\delta=-2^{\circ} 30^{\prime}$ in two

${ }^{4}$ http://www.sdss.org/dr6/tutorials/retrieveFITS.html

${ }^{5} \mathrm{http}: / /$ montage.ipac.caltech.edu/ complete samples: galaxies with angular diameters $>1^{\prime}$ on the first POSS blue prints and galaxies brighter than $m_{p}=14.5$ in the Zwicky Catalog of Galaxies and Clusters of Galaxies. The IRAS fluxes were obtained from $60 \mu \mathrm{m}$ and $100 \mu \mathrm{m}$ flux densities listed in either the IRAS Cataloged Galaxies and Quasars (Fullmer \& Londsdale, 1989) or the IRAS Faint Source Catalog v2.0 (Moshir et al., $1993)$ using the formula $f_{\mathrm{FIR}} / 10^{-11}=3.25 S_{60}+1.26 S_{100}$ following Ho et al. (1997) where $S_{60}$ and $S_{100}$ are the total flux densities at 60 and $100 \mu \mathrm{m}$, respectively. The IRAS catalogs are complete to approximately 1.5 Jy for pointlike sources. The combined selection criteria favor nearby $(D<15 \mathrm{Mpc})$ predominately optically-bright galaxies with at least a modest amount of recent star formation as inferred from their FIR luminosities. The sample is known to exclude smaller dwarf galaxies in the neighborhood (Swartz et al., 2008).

Eighty-nine of the 140 galaxies comprising the Xray sample are within the footprint of the 6th data release of the Sloan Digitized Sky Survey (Adelman-McCarthy et al., 2008). Of these, only the 58 galaxies with inclination $i<65^{\circ}$ make up the present study as it is too difficult to isolate local features in higher inclination galaxies.

Table 1 lists relevant properties of the galaxies included in this study. Distances are adopted from the Nearby Galaxies Catalog (Tully, 1988) augmented by those computed using the heliocentric velocities listed in the NASA/IPAC Extragalactic Database (NED) assuming $H_{o}=73 \mathrm{~km} \mathrm{~s}^{-1} \mathrm{Mpc}^{-1}$. Galaxy celestial coordinates were taken from the NED. Inclinations, optical shape $\left(D_{25}\right.$ : major isophotal diameter measured at surface brightness level of $25 \mathrm{mag} \mathrm{s}^{-2}$ in $B$, position angle, and major-to-minor axis ratio) and morphological type are from the Third Reference Catalogue of Bright Galaxies (de Vaucouleurs et al., 1991) Table 1 is composed of two sections: The 27 low-inclination galaxies hosting one or more ULX and the 31 low-inclination galaxies without ULXs.

\subsection{The SDSS Data}

All galaxies in the present study are extended objects by definition. Therefore, SDSS pipeline products such as object lists are not of relevance here. Instead, we begin with corrected imaging frames and use these to produce color maps for our analysis. Details of this process are given in this subsection.

\subsubsection{Preparation of Color Maps}

Corrected frame images of the target galaxies in each of the $u, g, r$ and $i$ filters were retrieved from the SDSS archivet. These are the imaging frames with flat field, bias, cosmic ray, and pixel defect corrections applied. Each covers an approximately $13.5^{\prime} \times 9.8^{\prime}$ region of the sky. In several cases, one or more adjacent frames were needed to cover a target galaxy; however, each image frame was processed separately.

There are often slight offsets among the 4 filter frames of a given field. The Montage(v3.0) image mosaic toolkit 5 module mProjectPP was used to re-project the frames and 
module mSubimage was used to center and to crop a small border off of each frame. This ensured that the same sky coordinates were assigned to a given pixel in all 4 frames of a field. Checks against known object positions were made to confirm that reprojection did not compromise the astrometric accuracy of the frames. This is important when registering ULX positions obtained from X-ray data to the final color maps.

An estimate of the sky level was then made by constructing an object mask that includes the $D_{25}$ ellipse of the target galaxy, any bright objects elsewhere in the frame as determined by our source-finding software (Tennant, 2006), and a small border zone to include any artifacts introduced by the reprojection. The sky level was then defined as the average of the unmasked pixels in each frame (after properly accounting for the soft bias and converting pixel data numbers to true counts using the gain parameters provided in the accompanying field information files).

Sky-subtracted corrected-frame data numbers were then converted to calibrated magnitudes using the zero points, extinction coefficients and airmass values also provided in the field information files. Dereddening to correct for Galactic extinction was also applied using the Hi maps of Kalberla et al. (2005) accessed through the FTOOL utility $\mathrm{nh}$ and applying the extinction conversion for each SDSS filter as given in the early data release paper (Stoughton et al., 2002).

Finally, $u-g, g-r$, and $r-i$ color maps were constructed by subtracting the appropriate magnitude image in one spectral band from that in another.

\subsubsection{Defining Local Regions}

Local regions are defined here to be the $100 \times 100 \mathrm{pc}^{2}$ area centered on the X-ray position of the ULX. This physical size was chosen to be roughly that of a moderate-sized $\mathrm{H}$ II region. For comparison, the average diameter for the $248 \mathrm{H}$ II regions in M 101 identified by Scowen et al. (1992) is $140 \mathrm{pc}$ and the average half-light diameter of the $492 \mathrm{H} \mathrm{II}$ regions in M 81 compiled by Petit et al. (1988) is 56 pc. Similarly, OB stellar associations in Local Group galaxies are typically 80-100 pc diameter (e.g., Hunter, 1995; Gouliermis et al., 2003).

The colors of local regions are defined as the average of the colors of all pixels within the region. Note this is not equivalent to the color derived from the difference of the average magnitudes in two spectral bands, i.e., it is not a flux-weighted but an area-weighted average color. In this way, small bright objects are not overemphasized. Our averaging method results in colors in units of mag per $100 \times 100 \mathrm{pc}^{2}$ region although, henceforth, color values in this work will be quoted simply in magnitudes with the fixed area implied. Note this "aperture photometry" also differs from that of isolated point-like objects for which a local background can be defined using a nearby or surrounding source-free region. Here, neighboring regions contain similar sources of emission and should not be considered a background. Thus, only the mean sky values are considered background in this work.

The $100 \times 100 \mathrm{pc}^{2}$ region, or resolution element, varies from $1.4^{\prime \prime} \times 1.4^{\prime \prime}\left(3.6 \times 3.6\right.$ pixels subtending $\left.0.396 \mathrm{~s} \mathrm{pixel}^{-1}\right)$ for the most distant galaxy in our sample up to $9.8^{\prime \prime} \times 9.8^{\prime \prime}$ $(24 \times 24$ pixels $)$ for the nearest target. In order to maintain consistency when comparing local regions hosting ULXs to whole galaxies, the same procedure is applied to all $100 \times 100 \mathrm{pc}^{2}$ regions within the $D_{25}$ area of each galaxy (excluding those $<3 \sigma$ above the sky level). We report the resulting average galaxy colors which are area-weighted colors defined as the sum over the colors of all resolution elements within the $D_{25}$ ellipse of a galaxy, divided by the number of elements. Average galaxy colors are listed in Table 1. For reference, the number of resolution elements range from 334 for the smallest galaxy (UGC 6850) to 53262 for the largest (M 101) in our sample.

\subsection{The X-ray Data: ULX Candidates}

The X-ray data for this study includes pointed observations obtained using Chandra, XMM-Newton, and ROSAT. The source-finding and characterization program described in Tennant (2006) was used to locate potential (point-like) ULXs. Source positions were compared to the NED coordinates of the host galaxy nucleus to cull potential AGN from the ULX list. These are the subject of another study (Zhang et al., 2009). Similarly, comparison was made to catalogs of sources to identify and cull known background galaxies and foreground stars. Furthermore, visual inspection of the SDSS images was made to identify potential interlopers not designated elsewhere. (This final inspection resulted in the rejection of one foreground star; no known background sources were found coincident with ULX candidate positions.) Applying the analytical form of the X-ray background $\log N-\log S$ distribution complied by Moretti et al. (2003) to the $D_{25}$ areas of each of the 58 galaxies in our sample, we expect a total of 1.3 background sources bright enough to be classified as ULXs. This number is small because the typical angular size of our sample galaxies is modest and the galaxies are relatively nearby so that ULXs sample only the sparsely-populated high-flux portion of the background $\log N-\log S$ distribution.

Forty-seven ULX candidates were identified in 27 of the 58 low-inclination galaxies in our sample. Their basic properties are listed in Table 2. All ULX candidates were observed with Chandra's ACIS detector and these data are used to characterize their X-ray porperties. Some of the (Chandra) observations are deep enough that the luminosities of ULX candidates can be accurately estimated using spectral fitting (taken from the literature or performed here following the methods of Swartz et al., 2004). Other observations are short exposures that accumulated only 20-50 events per ULX. For these short Chandra exposures, a straight counts-to-flux conversion is applied to estimate source luminosities as indicated in Table 2. The conversion assumes a power-law source spectrum of photon index 1.8 (Swartz et al., 2004) absorbed by the Galactic hydrogen column density along the line of site (from Kalberla et al., 2005). The conversion is roughly $f_{\mathrm{X}} \sim 10^{-11} \dot{N} \mathrm{ergs} \mathrm{cm}^{-2} \mathrm{~s}^{-1}$ where $f_{\mathrm{X}}$ is the flux and $\dot{N}$ is the observed count rate of events in the $0.5-8.0 \mathrm{keV}$ band. The exact conversion depends on the line-of-sight absorption. This counts-to-flux conversion was also applied to the deep Chandra observations to test the validity of the method; the simple estimates agree with published luminosities, where available, to within a factor of four which is adequate for our present purposes.

\section{STAR-FORMATION COLOR CRITERIA}


Two methods are employed to differentiate between the young stellar component and the older disk and bulge population in galaxies based on their SDSS colors. The first (33.1) is a simple empirical method that selects regions bluer in all three colors than $\mathrm{H}$ II regions. The second (\$3.2) is a more rigorous analytical method that finds the linear combination of colors which best separate starforming from non-star-forming regions. The two methods give very similar results.

\subsection{Empirical Star-Formation Criteria}

Here we establish, empirically, the SDSS colors of regions known by independent means to be sites of recent star formation. For nearby galaxies, an appropriate and easily-accessible recent star formation indicator is $\mathrm{H} \alpha$ emission from $\mathrm{H}$ II regions. This $\mathrm{H} \alpha$ emission comes from recombination of gas photoionized by massive $\mathrm{O}$ and early $\mathrm{B}$ stars and therefore indicates ages less than $\sim 10$ Myr. These stars themselves emit strongly in blue light. We therefore expect $\mathrm{H}$ II regions to be bluer than non-starforming regions in the broad SDSS filters. There are, however, two factors that could lead to redder colors; dust obscuration and the underlying stellar continuum. Thus, for instance, a low-luminosity $\mathrm{H}$ II region located within the bright bulge of a galaxy can appear more red than a similar region located in the disk.

To develop an emprical star-formation criteria, we examine two well-studied large galaxies in our sample; the early-type SA(s)ab spiral M 81 (NGC 3031) and the latetype SAB(rs)cd galaxy M 101 (NGC 5457). The top three panels of Figure 1 show the distribution of colors in the central bulge, disk, and cataloged H II regions (whose locations and angular sizes are taken from Petit et al., 1988) of M 81 in each of the three colors $u-g, g-r$, and $r-i$. The average of the colors of the $\mathrm{H}$ II regions and of the colors of the galaxy as a whole are listed in Table 3 (where errors denote one standard deviation). As expected, the $\mathrm{H}$ II regions are bluer than the bulge and the disk but there is considerable scatter which is due in part to obscuration by dust.

We performed a similar analysis for M 101 (bottom panels of Figure 1 and Table 3). We find, first of all, that the mean colors of the galaxy are bluer than those of M 81 due to the large contribution to the average colors from the (red) bulge of M81 in contrast to the (blue) diskdominated late-type galaxy M 101. The means of the colors of $\mathrm{H}$ II regions (sizes and locations from the tabulation of Scowen et al., 1992) are also bluer than those of M 81 (Table 3). This indicates that the SDSS colors of starforming regions are, indeed, determined in part by the underlying stellar light contribution and will vary from galaxy to galaxy.

To summarize, we may formulate our criterion to distinguish star-forming regions based on the following empirical evidence: (1) Star-forming regions are bluer in all three SDSS colors, in general, than are non-star-forming regions in the same galaxy. (2) Allowance must be made for reddening due to dust. (3) Allowance must be made for differences among galaxies due to differences in their underlying stellar continua. (4) Allowance must also be made for low $S / N$ regions for which colors are poorly constrained.

Thus, we adopt the following criterion to distinguish star-forming from non-star-forming regions. The mean colors of the M $81 \mathrm{HII}$ regions define the baseline. These values are scaled upward by $1 \sigma$ to allow for dust reddening. For each host galaxy, these values are scaled further by the difference of the mean colors of the host galaxy to that of M 81 to allow for variations in the underlying stellar continua. Symbolically, a region in the $i^{t h}$ galaxy is flagged as a star-forming region if, for all three colors $C_{j, i}$, $j=1,3$, of the region

$$
C_{j, i}<\left(h_{j}+\delta h_{j}\right)-\left(\left\langle C_{j, \mathrm{M} 81}\right\rangle-\left\langle C_{j, i}\right\rangle\right)
$$

where $h_{j}$ is the mean color and $\delta h_{j}$ is the standard deviation for $\mathrm{H}$ II regions in $\mathrm{M} 81,\left\langle C_{j, \mathrm{M} 81}\right\rangle$ is the average color of all $100 \times 100 \mathrm{pc}^{2}$ regions in M 81, and $\left\langle C_{j, i}\right\rangle$ is the average color of regions in the $i^{\text {th }}$ galaxy. In addition, regions fainter than $3 \sigma$ above the mean sky level in any of the four filters are rejected as low $S / N$ regions under criterion (4) above.

Applying Equation 11 to M 101 results in color criteria of $C_{j, \mathrm{M} 101}<1.07,0.50$, and $0.27 \mathrm{mag}$ for $u-g, g-r$, and $r-i$, respectively. These are close to the average colors (plus $1 \sigma$ ) of the known H II regions of this galaxy (Table 3). Table 1 (see $\S 2.1 .2$ ) includes the mean galaxy colors, $\left\langle C_{j, i}\right\rangle$, and the fraction, $f_{\mathrm{HII}}$, of $100 \times 100 \mathrm{pc}^{2}$ regions classified as bluer than typical $\mathrm{H}$ II regions according to Equation 1 for each galaxy in the sample.

\subsection{Fisher's Linear Discriminant}

The empirical criterion, Equation 1, treats the 3 SDSS colors as independent quantities. The possibility exists that some other combination of colors or magnitudes may better distinguish star-forming regions from other regions. To test this hypothesis, we used the "Toolkit for Multivariate Data Analysis with ROOT" or TMVA6, an addon package to the script interpreter ROOT available from CERN7. This Toolkit provides several methods of multivariate analysis to separate classes of objects. In applying the evaluation and test phases of the Toolkit, we found that linear discriminant analysis, in the form first described by Fisher (1936), performed very well and we adopted it here. The Fisher algorithm finds the linear combination of attributes that best separates two or more classes of objects. In our case, the attributes are the SDSS magnitudes and colors and we wish to distinguish the class of star-forming regions from the class of non-star-forming regions. The algorithm maximizes the ratio of the distance between the classes to the variance within the classes thereby guaranteeing maximal separability. The output is a set of linear coefficients, $a_{i}$ for the input attributes, $x_{i}$ that gives the Fisher index, $F=a_{0}+a_{1} x_{1}+a_{2} x_{2}+\cdots$, such that, in our case, $F>0$ would denote a star-forming region.

We began with the known class of $\mathrm{H}$ II regions in M 81 as the training set "source" and all $100 \times 100 \mathrm{pc}^{2}$ regions in M 81 (that are $>3 \sigma$ above the sky level) as the training set "background". Inspection of the resulting Fisher

\footnotetext{
${ }^{6}$ http://tmva.sourceforge.net/
}

${ }^{7}$ http://root.cern.ch/ 
index indicated that SDSS colors were more important attributes than magnitudes for separating star-forming from non-star-forming regions; which makes sense physically. Furthermore, there appeared to be little benefit gained by including all three colors with $g-r$ being the least important. This naturally suggested using just the two attributes, $u-g$ and $r-i$. Retraining using only these two attributes results in a Fisher index

$$
F=0.374-0.179(u-g)-0.491(r-i)
$$

Equation 2 did a very good job of separating young star forming regions in M 81 and provides a criterion comparable to the empirical method developed in 3.1

Applying Equation 2 to M 101 classifies most of the galaxy as star-forming because, as before, the mean colors of the early-type galaxy M 81 are redder than those of M 101. This is shown graphically in Figure 2. The left panel shows the distribution of M 101 regions using the H II regions of M 81 as the training set (Equation 2). The right panel shows the distribution resulting after retraining using the HII regions of M 101 itself. Both clearly separate the star-forming $\mathrm{H}$ II regions from non-star-forming regions although the distributions differ in several respects. The most important difference is the overall shift or offset from the fiducial $F=0$ separation criterion to $F \approx 0.1$ when using the M 81 training set. Noting that the average Fisher index for M 81 (computed using the average colors tabulated in Table 1 to evaluate Equation 2) is -0.036 and that for M 101 is +0.074 suggests that the Fisher index can be easily compensated for differences in the underlying galaxy colors by applying an offset $\Delta_{i}=\left(\left\langle F_{i}\right\rangle-\left\langle F_{\mathrm{M} 81}\right\rangle\right)$ such that a region in the $i^{\text {th }}$ galaxy is flagged as a star-forming region if $F>\Delta_{i}$. Here, $\left\langle F_{i}\right\rangle$ is the value of $F$ from Equation 2 evaluated using the average $u-g$ and $r-i$ colors of the $i^{t h}$ galaxy and $\left\langle F_{\mathrm{M} 81}\right\rangle$ is the corresponding value for M 81; $\left\langle F_{\mathrm{M} 81}\right\rangle=-0.036$. This results in $\Delta=0.109$ for M 101. This is wholly equivalent to the scaling by the mean galaxy colors that is part of the empirical criterion (item 3) leading to Equation 1.

A comparison of the two methods is given by the fraction of $\mathrm{H}$ II regions actually selected as star-forming. These are listed in Table 3 for M 81 and M 101.

Finally, a direct comparison of the two methods is illustrated in Figure 3, It is a color-color diagram for the M 81 training data. Each point represents the $r-i$ vs. $g-r$ colors of a $100 \times 100 \mathrm{pc}^{2}$ region in the galaxy with the known H II regions (from Petit et al., 1988) highlighted. The diagonal line traces $F=0$ from Equation 2 and the horizontal and vertical lines represent two of the three empirical cuts from Equation 1 and Table 3. Regions to the lower left in the figure are designated blue starforming regions according to these critiria. Note that the colors of M 81 regions at the red portion of the diagram, $(u-g, r-i) \approx(1.8,0.5)$, correspond to those of K0 main sequence stars, as expected, whereas those in the blue portion, say $(u-g, r-i) \approx(1.0,0.3)$, do not correspond to colors of a single stellar population; $u-g=1.0$ corresponds to B8 to A0 stars whereas $r-i=0.3$ is the color of G0 to G5 stars. This is further evidence of the contribution to young star-forming regions of the older underlying disk and bulge populations.

\section{RESULTS}

The two criteria developed in the previous section provide similar results. We emphasize results from the empirical method, 3.1, in the following but also quote values from the Fisher linear discriminant when appropriate.

\subsection{Quantitative Considerations}

We can divide the $100 \times 100 \mathrm{pc}^{2}$ regions surrounding the 47 ULX candidates in our sample into three nonoverlapping groups: Those rejected as being too faint for quantitative analysis of their colors; those meeting the star-forming criterion; and those that do not meet the star-forming criterion. For brevity, these groups will be designated as $R$ for rejected, $S$ for star-forming, and $N$ for non-star-forming, respectively; they are listed in Table 2. There are 12 ULX candidates in $R$ regions. Using the empirical criterion, there are 21 ULX candidates in $S$ regions and 14 in $N$ regions. The results are similar for the Fisher criterion except that 6 regions identified as $S$ by Equation 1 are classified as non-starforming by the Fisher criterion and 2 regions identified as $N$ are classified as star-forming. Inspection of the SDSS 3-filter images 8 did not suggest a reason for this discrepancy. However, we note that the two methods agree well in the statistical sense.

As a simple check against the possibility of introducing biases into our analysis due to large-scale structures within ULX host galaxies, we queried a control sample of regions in each galaxy and analyzed them in a manner completely analogous to that of regions surrounding the ULX candidates. This sample is defined as the $100 \times 100 \mathrm{pc}^{2}$ regions diametrically opposed to the ULX regions in each host galaxy. In this way, at least for well-ordered spirals, the control group samples regions similar to those of the ULXs on large scales but not on the small scales of individual star-forming regions. Using the same group definitions as given above for the ULX population, the control sample is distributed as 21(21) group $R$ objects, $7(0)$ group $S$ objects, and 19(26) group $N$ objects using the empirical (Fisher) criterion. This indicates the ULXs are preferentially located in star-forming regions and that these regions are more luminous than their surroundings.

We can also estimate the probability of a random sampling of star-forming regions. We applied the empirical star-formation criteria to all $100 \times 100 \mathrm{pc}^{2}$ regions in the 27 galaxies containing ULXs. This ranks $31.8 \%$ of all regions bright enough for analysis as star-forming. Thus if ULXs occurred randomly, we would expect only 11 of the 35 to be classified as star forming regions. The chance of finding 21 when only 11 are expected is a $3.1 \sigma$ deviation. On the other hand, finding that 7 of 26 control sample regions, or $27 \%$, are star-forming is wholly consistent with a random distribution of control sample regions. The more restrictive Fisher criterion classifies only $23.5 \%$ of regions as star-forming. The chance of finding 17 ULXs in starforming regions when only 8 are expected is a $3.1 \sigma$ deviation while finding 0 of the control sample in star-forming regions when 5 are expected is $2.3 \sigma$ low.

\subsubsection{Correlations with Star-forming Regions}

${ }^{8}$ available on-line from http://cas.sdss.org/astro/en/tools/chart/chart.asp 
Fully $60 \%$ of those ULX regions with sufficient signal $(21 / 35)$ are in star-forming regions. By definition, these regions are as blue or bluer than typical Hil regions of their host galaxies and therefore are likely of an age also typical of $\mathrm{H}$ II regions which is $\$ 10 \mathrm{Myr}$; the characteristic lifetime of the least-massive LyC-producing stars, i.e., late $\mathrm{O}$ to early $\mathrm{B}$ stars of about 15 to $20 \mathrm{M}_{\odot}$.

\subsubsection{Correlations with Host Galaxy Morphology}

Ten of the 27 galaxies hosting ULX candidates in our sample are early-type galaxies (S0 through $\mathrm{Sbc}$ ). There is no preference for objects in any of the 3 groups, $R, S$, or $N$, to be associated with a particular galaxy morphological type.

\subsubsection{Correlations with ULX Luminosity}

The average X-ray luminosity of group $S$ objects is only $1.7 \pm 0.7$ in units of $10^{39} \mathrm{ergs} \mathrm{s}^{-1}$. The average of group $N$ is $3.5 \pm 3.6$ and of group $R$ is $3.9 \pm 3.8$. These numbers reflect the fact that most (74\%) of the ULXs in the current sample have observed luminosities estimated to be $<3 \times 10^{39} \mathrm{ergs} \mathrm{s}^{-1}$ and that 10 of those 12 more luminous than this value are equally divided among groups $N$ and $R$. Thus, the average luminosities in these groups are high while the scatter in their luminosities is large.

\subsubsection{Comparison to Mean Galaxy Colors}

A comparison of the color distributions of all $100 \times 100 \mathrm{pc}^{2}$ regions in all ULX host galaxies with the color distribution of ULX regions and with the color distribution of the control sample is shown in Figure 4. The three panels present this comparison for the three colors $u-g, g-r$, and $r-i$. In each panel, the contributions from each galaxy are shifted along the abscissa such that regions that exactly meet the color criterion of Equation 1 have a color of zero. For clarity of presentation, the histograms representing the contributions from all regions (solid histograms) are normalized to 100 . Only resolution elements brighter than $3 \sigma$ above the mean sky level, including those containing ULXs and the control sample, are included in this census.

Also shown, in each panel, are best-fitting Gaussian functions fitted to each distribution. The best-fit parameter values are listed in Table 4 .

The ULX $u-g$ color distribution is significantly bluer than that of their host galaxies and of the control sample. The ULX color distributions in $g-r$ and $r-i$ are similar to their host galaxies but still bluer than the control sample. The ULX color distributions are broad in all 3 colors indicating the range of environments in which they are found from red galaxy bulges and dust lanes to blue young star forming regions. The trends also show, again, the strong dependence on the underlying stellar population to the determination of colors of individual regions.

\subsection{Qualitative considerations}

We inspected our color maps and the on-line SDSS images and consulted the literature for additional information on the optical properties of the environments of the ULXs in our sample. We checked whether some of the group $N$ objects may be star-forming regions that are heavily reddened by dust either local to the region or superimposed along the line of sight. Only two of the 14 group $N$ regions can be confidently associated with strong dust lanes based on the morphology of their colors. The Xray luminosities of these two objects are among the highest in the sample: 11.1 and $11.3 \times 10^{39} \mathrm{ergs} \mathrm{s}^{-1}$. Visual inspection shows that 5 other group $N$ regions are clearly within the bulges of their respective hosts. Six others appear to be in disk regions near to but not coincident with blue star clusters. These are designated "patchy blue" in Table 2 along with several of the group $R$ objects. (The remaining group $N$ region appears to be a bright star cluster. Its colors are inconsistent with those of individual stars and of background galaxies. It is designated "star cluster?" in Table 2.) Thus, at most only a few of the ULXs in group $N$ can possibly be reddened group $S$ objects. We also note that galaxies hosting group $N$ regions are not systematically stronger (dust-rich) FIR emitters than are group $S$ hosts. Neither are they more inclined to our line-of-sight than are group $S$ hosts. (Of course, some galaxies host both group $N$ and group $S$ objects).

Visual inspections also showed four ULX regions with relatively strong emission in the $r$ filter. Two of these are designated group $R$ and two are group $N$ regions with $g-r$ colors redder than typical of their host galaxy's H II regions. Lacking supporting evidence at this time, we speculate that these regions are isolated $\mathrm{H}$ II regions whose $r$-band emission is dominated by $\mathrm{H} \alpha$ with only weak contributions from underlying starlight. These are denoted by "H $\alpha$ ?" in the Comments column of Table 2.

\section{DISCUSSION}

We have devised simple criteria, calibrated on the SDSS optical colors of known H II regions in M 81 and M 101, to distinguish young, star-forming regions in nearby galaxies. We have used these criteria to examine the $100 \times 100 \mathrm{pc}^{2}$ regions around ULX candidates to determine if they are associated with the young stellar population. ULX environments are designated as "star-forming" if they are as blue as, or bluer than a typical H II region in that galaxy (after taking also into account the reddening effects of dust and of old underlying stellar populations by scaling by the mean colors of that galaxy relative to M 81). A useful feature of this method is that colors are distance-independent quantities, and are measured on distance-independent regions.

For the ULX regions bright enough to permit a quantitative analysis of their colors, we found that $60 \%$ are bluer than their host galaxy's H II regions in all three SDSS colors. This indicates a significant correlation between these ULXs and young OB associations. However, none of them is associated with a compact super-star-cluster within a $100 \times 100 \mathrm{pc}^{2}$ region. This is at odds with models that invoke O-star collision and merger processes in the collapsed core of a super-star-cluster (Portegies Zwart et al., 2004; Freitag et al., 2006), leading to the formation of an IMBH powering the ULX. It is possible that some ULXs do indeed form this way but this does not appear to be, by any measure, a common formation mechanism. Conversely, there are several ULXs located in the bulges of early-type spirals. We interpret them as members of a second, low-mass-donor, population in contrast with the high-mass systems associated with recent and on-going star formation.

Between these two extremes are several ULXs near 
patchy blue regions of local star formation. At the same time, we found that 10 out of the 12 most luminous ULXs in our sample (those with $L_{\mathrm{X}} \gtrsim 3 \times 10^{39} \mathrm{ergs} \mathrm{s}^{-1}$ ) are located in regions classified as faint or non-starforming. Perhaps a couple of these luminous ULX regions are heavily reddened by dust. Note that the probability of finding a ULX outside a star-forming region is 0.5532 (26 of 47 ULXs are classified as either $R$ or $N$ ) therefore the probability of getting 10 or more non- $S$ regions in 12 trials selected at random from our sample is $4.4 \%$.

For a more physical interpretation, we recall that our calibrator for star formation is the optical color distribution of Hil regions. We speculate that the most luminous ULXs (or equivalently, the most common phases of very high mass transfer) are biased towards early B-type donors with an initial mass of $\approx 10-15 \mathrm{M}_{\odot}$ and an age $\sim 10-20$ Myr (perhaps at the stage where the B star expands to become a blue supergiant). In that case, we expect very little residual $\mathrm{H} \alpha$ emission from their surroundings, the $\mathrm{O}$ stars having already evolved and died. The ULX region in this scenario will appear redder than surrounding $\mathrm{H}$ II regions where star formation is still ongoing. Individual studies of stellar populations around some luminous ULXs are consistent with this explanation: for example, an age $\approx 10-20 \mathrm{Myr}$ was inferred for the stars around NGC 4559 X-1 (Soria et al., 2005), and $\approx 20 \mathrm{Myr}$ for those around NGC 1313 X-2 (Grisé et al., 2008).

Another interpretation of the observed separation between luminous X-ray sources and nearby star-forming regions (Zezas et al., 2002; Kaaret et al., 2004) is that X-ray binaries are expelled from their place of birth. This can occur through a variety of processes such as an asymmetric supernova that forms the compact remnant of the binary or interactions between the binary and other stars in the cluster (see Kaaret et al., 2004, for further discussion). Typical runaway velocities are of order $10 \mathrm{~km} \mathrm{~s}^{-1}$ and typical separations between X-ray binaries and star clusters are $\sim 200 \mathrm{pc}$ implying ages of $\sim 20$ Myrs. However, Kaaret et al. (2004) also find the cluster-binary separation is shorter for more luminous $\mathrm{X}$-ray sources suggesting a higher system mass (lower kick velocity) for these objects. This is inconsistent with the general pattern described here where 10 of the 12 most luminous ULXs are further from star-forming regions than are the majority of fainter ULXs.

The possibility that young, relatively high-mass, systems may be located outside well-defined star-forming regions implies there remains an observational degeneracy between the $\$ 20$ Myr-old high-mass-donor and the Gyrold low-mass-donor ULX populations. This degeneracy is compounded by the reverse scenario; an old low-mass system may be located within, or along the line-of-sight towards, a star-forming region.

However, in a more general sense, we have shown that regions of nearby galaxies with differing recent starformation histories can be separated using SDSS colors. Hopefully, this method can be applied more generally to identify and study correlations between classes of sources with stellar populations or substructures in nearby galaxies.

This work was supported, in part, by Chandra Award GO6-7081A issued by the Chandra X-ray Observatory Center which is operated by the Smithsonian Astrophysical Observatory for and on behalf of NASA under contract NAS8-03060. This work has made extensive use of DR6 of the Sloan Digitized Sky Survey. Funding for the SDSS and SDSS-II has been provided by the Alfred P. Sloan Foundation, the Participating Institutions, the National Science Foundation, the U.S. Department of Energy, the National Aeronautics and Space Administration, the Japanese Monbukagakusho, the Max Planck Society, and the Higher Education Funding Council for England.

References

Adelman-McCarthy, J. K. et al. 2008, ApJS, 175, 297

Baldry, I. K., Glazebrook, K., Brinkmann, J., Ivezić, Ž., Lupton, R. H., Nichol, R. C., \& Szalay, A. S. 2004, ApJ, 600, 681

Bruzual, G. 1983, ApJ, 273, 105

de Vaucouleurs, G., de Vaucouleurs, A., Corwin, Jr., H. G., Buta, R. J., Paturel, G., \& Fouque, P. 1991, Third Reference Catalogue of Bright Galaxies (Springer-Verlag Berlin Heidelberg New York)

Elmegreen, B. G. 2004, MNRAS, 354, 367

Elmegreen, B. G., \& Efremov, Y. N. 1997, ApJ, 480, 235

Fisher, R. A. 1936, Annals of Eugenics, 7, 179

Freitag, M., Gürkan, M. A., \& Rasio, F. A. 2006, MNRAS, 368, 141

Fullmer, L., \& Londsdale, C. J. 1989, Cataloged galaxies and quasars observed in the IRAS survey (Pasadena: Jet Propulsion Laboratory, Version 2)

Gilfanov, M., Grimm, H.-J., \& Sunyaev, R. 2004, MNRAS, 347, L57

Gouliermis, D., Kontizas, M., Kontizas, E., \& Korakitis, R. 2003, A\&A, 405, 111

Grisé, F., Pakull, M. W., Soria, R., Motch, C., Smith, I. A., Ryder, S. D., \& Böttcher, M. 2008, A\&A, 486, 151

Heger, A., Fryer, C. L., Woosley, S. E., Langer, N., \& Hartmann, D. H. 2003, ApJ, 591, 288 
Heger, A., \& Woosley, S. E. 2002, ApJ, 567, 532

Ho, L. C., Filippenko, A. V., \& Sargent, W. L. W. 1997, ApJS, 112, 315

Hunter, D. A. 1995, in Revista Mexicana de Astronomia y Astrofisica Conference Series, Vol. 3, Revista Mexicana de Astronomia y Astrofisica Conference Series, ed. M. Pena \& S. Kurtz, 1-+

Kaaret, P., Alonso-Herrero, A., Gallagher, J. S., Fabbiano, G., Zezas, A., \& Rieke, M. J. 2004, MNRAS, 348, L28

Kalberla, P. M. W., Burton, W. B., Hartmann, D., Arnal, E. M., Bajaja, E., Morras, R., \& Pöppel, W. G. L. 2005, A\&A, 440,775

Kauffmann, G. et al. 2003, MNRAS, 341, 33

Kuntz, K. D., Gruendl, R. A., Chu, Y.-H., Chen, C.-H. R., Still, M., Mukai, K., \& Mushotzky, R. F. 2005, ApJ, 620, L31

Liu, J.-F., Bregman, J. N., \& Irwin, J. 2006, ApJ, 642, 171

Liu, J.-F., Bregman, J. N., \& Seitzer, P. 2002, ApJ, 580, L31

-. 2004, ApJ, 602, 249

Moretti, A., Campana, S., Lazzati, D., \& Tagliaferri, G. 2003, ApJ, 588, 696

Moshir, M. et al. 1993, VizieR Online Data Catalog, 2156, 0

Nilson, P. 1973, Uppsala general catalogue of galaxies (Acta Universitatis Upsaliensis. Nova Acta Regiae Societatis Scientiarum Upsaliensis - Uppsala Astronomiska Observatoriums Annaler, Uppsala: Astronomiska Observatorium, 1973)

Ohkubo, T., Umeda, H., Maeda, K., Nomoto, K., Suzuki, T., Tsuruta, S., \& Rees, M. J. 2006, ApJ, 645, 1352

Pakull, M. W., Grisé, F., \& Motch, C. 2006, in IAU Symposium, Vol. 230, Populations of High Energy Sources in Galaxies, ed. E. J. A. Meurs \& G. Fabbiano, 293-297

Pakull, M. W., \& Mirioni, L. 2002, ArXiv Astrophysics e-prints

Petit, H., Sivan, J.-P., \& Karachentsev, I. D. 1988, A\&AS, 74, 475

Portegies Zwart, S. F., Baumgardt, H., Hut, P., Makino, J., \& McMillan, S. L. W. 2004, Nature, 428, 724

Ptak, A., Colbert, E., van der Marel, R. P., Roye, E., Heckman, T., \& Towne, B. 2006, ApJS, 166, 154

Roberts, T. P., Levan, A. J., \& Goad, M. R. 2008, MNRAS, 387, 73

Scowen, P. A., Dufour, R. J., \& Hester, J. J. 1992, AJ, 104, 92

Soria, R., Cropper, M., Pakull, M., Mushotzky, R., \& Wu, K. 2005, MNRAS, 356, 12

Stoughton, C., Lupton, R. H., Bernardi, M., Blanton, M. R., Burles, S., Castander, F. J., \& Connolly, A. J. 2002, AJ, 123,485

Strateva, I. e. a. 2001, AJ, 122, 1861

Swartz, D. A., Ghosh, K. K., Tennant, A. F., \& Wu, K. 2004, ApJS, 154, 519

Swartz, D. A., Soria, R., \& Tennant, A. F. 2008, ApJ, 684, 282

Tennant, A. F. 2006, AJ, 132, 1372

Terashima, Y., Inoue, H., \& Wilson, A. S. 2006, ApJ, 645, 264

Tully, R. B. 1988, Nearby galaxies catalog (Cambridge, Cambridge University Press)

van der Marel, R. P. 2004, in Coevolution of Black Holes and Galaxies, ed. L. C. Ho, 37-+

Weidner, C., \& Kroupa, P. 2006, MNRAS, 365, 1333

Yungelson, L. R., van den Heuvel, E. P. J., Vink, J. S., Portegies Zwart, S. F., \& de Koter, A. 2008, A\&A, 477, 223

Zezas, A., Fabbiano, G., Rots, A. H., \& Murray, S. S. 2002, ApJ, 577, 710

Zhang, W. M., Soria, R., Zhang, S. N., Swartz, D. A., \& Liu, J. 2009, ArXiv e-prints 
TABLE 1

Properties of SDSS Galaxies

\begin{tabular}{|c|c|c|c|c|c|c|c|c|c|}
\hline Galaxy & $\begin{array}{c}\text { R.A. } \\
\text { (h m s) }\end{array}$ & $\begin{array}{c}\text { Decl. } \\
(\mathrm{d} \mathrm{m} \mathrm{s})\end{array}$ & Type & $\begin{array}{c}D \\
(\mathrm{Mpc})\end{array}$ & $\begin{array}{c}D_{25} \\
(\min )\end{array}$ & $\begin{array}{c}u-g \\
(\mathrm{mag})^{a}\end{array}$ & $\begin{array}{c}g-r \\
(\mathrm{mag})^{a}\end{array}$ & $\begin{array}{c}r-i \\
(\mathrm{mag})^{a}\end{array}$ & $f_{\mathrm{HII}}^{b}$ \\
\hline \multicolumn{10}{|c|}{ SDSS Galaxies inclined $<65^{\circ}$ hosting ULXs } \\
\hline NGC 1068 & 024240.7 & -000048 & (R)SA(rs)b & 14.4 & 7.1 & 1.24 & 0.59 & 0.31 & 0.36 \\
\hline NGC 2500 & 080153.2 & +504414 & $\mathrm{SB}(\mathrm{rs}) \mathrm{d}$ & 10.1 & 2.9 & 0.81 & 0.30 & 0.18 & 0.29 \\
\hline NGC 2541 & 081440.1 & +490341 & $\mathrm{SA}(\mathrm{s}) \mathrm{cd}$ & 10.6 & 6.3 & 0.59 & 0.33 & 0.13 & 0.18 \\
\hline NGC 2681 & 085332.7 & +511849 & (R')SAB(rs)0/a & 13.3 & 3.6 & 1.44 & 0.58 & 0.35 & 0.39 \\
\hline NGC 2841 & 092202.6 & +505835 & $\mathrm{SA}(\mathrm{r}) \mathrm{b}$ & 12.0 & 8.1 & 1.48 & 0.70 & 0.41 & 0.39 \\
\hline NGC 3031 & 095533.2 & +690355 & $\mathrm{SA}(\mathrm{s}) \mathrm{ab}$ & 3.6 & 26.9 & 1.30 & 0.63 & 0.36 & 0.38 \\
\hline NGC 3184 & $1018 \quad 17.0$ & +412528 & $\mathrm{SAB}(\mathrm{rs}) \mathrm{cd}$ & 8.7 & 7.4 & 1.13 & 0.47 & 0.27 & 0.33 \\
\hline NGC 3239 & 102504.9 & +170949 & $\mathrm{IB}(\mathrm{s}) \mathrm{m}(\mathrm{pec})$ & 8.1 & 5.0 & 0.67 & 0.23 & 0.31 & 0.23 \\
\hline NGC 3627 & 112015.0 & +125930 & $\mathrm{SAB}(\mathrm{s}) \mathrm{b}$ & 6.6 & 9.1 & 1.31 & 0.57 & 0.32 & 0.38 \\
\hline NGC 3675 & 112608.6 & +433509 & $\mathrm{SA}(\mathrm{s}) \mathrm{b}$ & 12.8 & 5.9 & 1.40 & 0.70 & 0.40 & 0.33 \\
\hline UGC 6850 & 115237.3 & -022810 & Pec & 14.5 & 0.6 & 0.47 & 0.12 & -0.13 & 0.23 \\
\hline NGC 4136 & 120917.7 & +295539 & $\mathrm{SAB}(\mathrm{r}) \mathrm{c}$ & 9.7 & 4.0 & 0.92 & 0.34 & 0.18 & 0.29 \\
\hline NGC 4150 & 121033.6 & +302406 & $\mathrm{SA}(\mathrm{r}) 0$ & 9.7 & 2.3 & 1.43 & 0.61 & 0.39 & 0.17 \\
\hline NGC 4204 & 121514.3 & +203932 & $\mathrm{SB}(\mathrm{s}) \mathrm{dm}$ & 7.9 & 3.6 & 0.64 & 0.36 & 0.18 & 0.31 \\
\hline NGC 4449 & 122811.9 & +440540 & $\mathrm{IBm}$ & 3.0 & 6.2 & 0.88 & 0.41 & 0.16 & 0.30 \\
\hline NGC 4490 & 123036.4 & +413837 & $\mathrm{SB}(\mathrm{s}) \mathrm{d}(\mathrm{pec})$ & 7.8 & 6.3 & 0.87 & 0.30 & 0.13 & 0.22 \\
\hline NGC 4559 & 123557.7 & +275735 & $\mathrm{SAB}(\mathrm{rs}) \mathrm{cd}$ & 9.7 & 10.7 & 0.91 & 0.35 & 0.21 & 0.26 \\
\hline NGC 4561 & 123608.1 & +19 1921 & $\mathrm{SB}(\mathrm{rs}) \mathrm{dm}$ & 12.3 & 1.5 & 0.80 & 0.22 & 0.13 & 0.34 \\
\hline NGC 4618 & 124132.8 & +410903 & $\mathrm{SB}(\mathrm{rs}) \mathrm{m}$ & 7.3 & 4.2 & 0.90 & 0.37 & 0.17 & 0.40 \\
\hline NGC 4625 & 124152.7 & +411625 & $\mathrm{SAB}(\mathrm{rs}) \mathrm{m}(\mathrm{pec})$ & 8.2 & 2.2 & 1.06 & 0.51 & 0.20 & 0.37 \\
\hline NGC 4725 & 125026.6 & +253003 & $\mathrm{SAB}(\mathrm{r}) \mathrm{ab}(\mathrm{pec})$ & 12.4 & 10.7 & 1.37 & 0.65 & 0.37 & 0.31 \\
\hline UGC 8041 & 125512.6 & +000700 & $\mathrm{SB}(\mathrm{s}) \mathrm{d}$ & 14.2 & 3.1 & 0.72 & 0.33 & 0.16 & 0.19 \\
\hline NGC 5055 & 131549.3 & +420145 & $\mathrm{SA}(\mathrm{rs}) \mathrm{bc}$ & 7.2 & 12.6 & 1.16 & 0.63 & 0.35 & 0.34 \\
\hline NGC 5204 & 132936.5 & +582507 & $\mathrm{SA}(\mathrm{s}) \mathrm{m}$ & 4.8 & 5.0 & 0.68 & 0.24 & 0.12 & 0.22 \\
\hline NGC 5457 & 140312.6 & +542057 & $\mathrm{SAB}(\mathrm{rs}) \mathrm{cd}$ & 5.4 & 28.8 & 1.04 & 0.42 & 0.23 & 0.21 \\
\hline NGC 5474 & 140501.6 & +533944 & $\mathrm{SA}(\mathrm{s}) \mathrm{cd}(\mathrm{pec})$ & 6.0 & 4.8 & 0.91 & 0.37 & 0.18 & 0.28 \\
\hline NGC 5585 & 141948.2 & +564345 & $\mathrm{SAB}(\mathrm{s}) \mathrm{d}$ & 7.0 & 5.7 & 0.76 & 0.27 & 0.16 & 0.16 \\
\hline
\end{tabular}

SDSS Galaxies inclined $<65^{\circ}$ not hosting ULXs

\begin{tabular}{|c|c|c|c|c|c|c|c|c|c|}
\hline NGC 14 & 000846.4 & +154856 & $(\mathrm{R}) \operatorname{IB}(\mathrm{s}) \mathrm{m}(\mathrm{pec})$ & 12.8 & 2.8 & 1.01 & 0.39 & 0.22 & 0.40 \\
\hline NGC 2537 & 081314.6 & +455923 & $\mathrm{SB}(\mathrm{s}) \mathrm{m}(\mathrm{pec})$ & 9.0 & 1.7 & 1.00 & 0.47 & 0.24 & 0.38 \\
\hline NGC 2903 & 093210.1 & +213003 & $\mathrm{SAB}(\mathrm{rs}) \mathrm{bc}$ & 6.3 & 12.6 & 1.18 & 0.53 & 0.33 & 0.32 \\
\hline NGC 2976 & $0947 \quad 15.4$ & +675459 & $\mathrm{SAc}(\mathrm{pec})$ & 2.1 & 5.9 & 1.26 & 0.52 & 0.28 & 0.35 \\
\hline NGC 3077 & 100319.1 & +684402 & I0(pec) & 2.1 & 5.4 & 1.47 & 0.64 & 0.28 & 0.34 \\
\hline NGC 3274 & 103217.3 & +274008 & $\mathrm{SABd}$ & 5.9 & 2.1 & 0.76 & 0.27 & 0.10 & 0.23 \\
\hline NGC 3319 & 103909.5 & +414113 & $\mathrm{SB}(\mathrm{rs}) \mathrm{cd}$ & 11.5 & 6.2 & 0.66 & 0.36 & 0.22 & 0.17 \\
\hline NGC 3344 & 104331.1 & +245520 & (R)SAB $\mathrm{SA}$ )bc & 6.1 & 7.1 & 1.01 & 0.42 & 0.30 & 0.23 \\
\hline NGC 3351 & 104357.7 & +114213 & $\mathrm{SB}(\mathrm{r}) \mathrm{b}$ & 8.1 & 7.4 & 1.28 & 0.61 & 0.30 & 0.37 \\
\hline NGC 3368 & 104645.7 & +114912 & $\mathrm{SAB}(\mathrm{rs}) \mathrm{ab}$ & 8.1 & 7.6 & 1.35 & 0.64 & 0.34 & 0.33 \\
\hline NGC 3486 & 110023.9 & +285829 & $\mathrm{SAB}(\mathrm{r}) \mathrm{c}$ & 7.4 & 7.1 & 0.82 & 0.34 & 0.22 & 0.14 \\
\hline NGC 3738 & 113548.8 & +543126 & $\mathrm{Im}$ & 4.3 & 2.5 & 1.13 & 0.41 & 0.18 & 0.27 \\
\hline NGC 3985 & 115642.1 & +482002 & $\mathrm{SB}(\mathrm{s}) \mathrm{m}$ & 8.3 & 1.3 & 1.00 & 0.45 & 0.25 & 0.47 \\
\hline NGC 4020 & 115856.9 & +302449 & SBd & 8.0 & 2.1 & 0.93 & 0.40 & 0.21 & 0.31 \\
\hline NGC 4062 & 120403.8 & +315345 & $\mathrm{SA}(\mathrm{s}) \mathrm{c}$ & 9.7 & 4.1 & 1.25 & 0.58 & 0.33 & 0.33 \\
\hline NGC 4203 & 121505.0 & +331150 & SABO- & 9.7 & 3.4 & 1.62 & 0.73 & 0.39 & 0.28 \\
\hline NGC 4207 & 121530.4 & +093506 & $\mathrm{~S}$ & 8.3 & 1.6 & 1.36 & 0.64 & 0.33 & 0.37 \\
\hline NGC 4214 & 121539.2 & +361937 & $\mathrm{IAB}(\mathrm{s}) \mathrm{m}$ & 3.5 & 8.5 & 0.88 & 0.34 & 0.20 & 0.23 \\
\hline NGC 4245 & 121736.8 & +293629 & $\mathrm{SB}(\mathrm{r}) 0 / \mathrm{a}$ & 9.7 & 2.9 & 1.53 & 0.68 & 0.38 & 0.36 \\
\hline NGC 4309 & 122212.4 & +070839 & $\mathrm{SAB}(\mathrm{r}) 0+$ & 11.9 & 1.8 & 1.37 & 0.68 & 0.35 & 0.31 \\
\hline NGC 4310 & 122226.3 & +291231 & (R')SAB $(r) 0+$ & 9.7 & 2.2 & 1.33 & 0.68 & 0.41 & 0.34 \\
\hline NGC 4314 & 122232.0 & +295343 & $\mathrm{SB}^{\prime}(\mathrm{rs}) \mathrm{a}$ & 9.7 & 4.2 & 1.55 & 0.71 & 0.38 & 0.28 \\
\hline NGC 4370 & 122454.9 & +072640 & $\mathrm{Sa}$ & 10.7 & 1.4 & 1.62 & 0.74 & 0.39 & 0.40 \\
\hline NGC 4395 & 122548.9 & +333248 & $\mathrm{SA}(\mathrm{s}) \mathrm{m}$ & 3.6 & 13.2 & 0.78 & 0.27 & 0.16 & 0.26 \\
\hline NGC 4414 & 122627.1 & +311325 & $\mathrm{SA}(\mathrm{rs}) \mathrm{c}$ & 9.7 & 3.6 & 1.24 & 0.58 & 0.33 & 0.39 \\
\hline NGC 4491 & 123057.1 & +112901 & $\mathrm{SB}(\mathrm{s}) \mathrm{a}$ & 6.8 & 1.7 & 1.41 & 0.65 & 0.34 & 0.37 \\
\hline NGC 4509 & 123306.8 & +320530 & $\mathrm{Sab}(\mathrm{pec})$ & 12.8 & 0.9 & 0.65 & 0.21 & 0.05 & 0.33 \\
\hline
\end{tabular}


TABLE 2

Properties of ULX Candidates

\begin{tabular}{|c|c|c|c|c|c|c|c|c|c|}
\hline $\begin{array}{c}\text { R.A. } \\
(\mathrm{h} \mathrm{m} \mathrm{s})\end{array}$ & $\begin{array}{c}\text { Decl. } \\
(\mathrm{d} \mathrm{m} \mathrm{s})\end{array}$ & $\begin{array}{c}\text { Galaxy } \\
\text { Host } \\
\end{array}$ & $\begin{array}{c}L_{\mathrm{X}}^{a} / 10^{39} \\
\left(\mathrm{ergs} \mathrm{s}^{-1}\right)\end{array}$ & $\begin{array}{l}u-g^{b} \\
(\mathrm{mag})\end{array}$ & $\begin{array}{l}g-r^{b} \\
(\mathrm{mag})\end{array}$ & $\begin{array}{l}r-i^{b} \\
(\mathrm{mag})\end{array}$ & $\sigma_{\min }^{c}$ & Group $^{d}$ & Comments \\
\hline $02 \quad 4238.89$ & $-00 \quad 0055.1$ & NGC1068 & $8.0(11.3)$ & 1.18 & 0.73 & 0.31 & 11.0 & $\mathrm{~N}$ & dust lane \\
\hline 024239.71 & -00 0101.4 & NGC1068 & $3.0(1.4)$ & 1.07 & 0.41 & 0.26 & 28.2 & $\mathrm{~S}$ & \\
\hline 024240.43 & -00 0052.6 & NGC1068 & $1.3(0.6)$ & 1.60 & 0.75 & 0.32 & 47.2 & $\mathrm{~N}$ & bulge \\
\hline 080148.10 & 504354.6 & NGC2500 & $6.0(\mathrm{c} 2 \mathrm{f})$ & 0.88 & 0.24 & 0.04 & 4.0 & $\mathrm{~N}(\mathrm{~S})$ & patchy blue \\
\hline 080157.85 & 504339.5 & NGC2500 & $1.2(\mathrm{c} 2 \mathrm{f})$ & 0.58 & 0.16 & 0.26 & 14.5 & $\mathrm{~N}$ & star cluster? \\
\hline 081437.02 & 490326.6 & NGC2541 & $4.4(\mathrm{c} 2 \mathrm{f})$ & 0.74 & 0.24 & 0.25 & 1.3 & $\mathrm{R}$ & patchy blue \\
\hline 085333.66 & 511929.5 & NGC2681 & $1.2(0.9)$ & 1.34 & 0.59 & 0.38 & 2.2 & $\mathrm{R}$ & \\
\hline 085335.75 & 511917.3 & NGC2681 & $0.7(1.8)$ & 1.06 & 0.65 & 0.38 & 3.3 & $\mathrm{~S}$ & \\
\hline 092202.22 & 505854.2 & NGC2841 & $1.1(0.7)$ & 1.81 & 0.87 & 0.47 & 13.3 & $\mathrm{~N}$ & bulge \\
\hline 095532.97 & 690033.4 & NGC3031 & $2.9(3.8)$ & 1.20 & 0.69 & 0.35 & 13.2 & $\mathrm{~S}(\mathrm{~N})$ & \\
\hline 101812.05 & 412420.7 & NGC3184 & $1.0(1.7)$ & 1.88 & 0.45 & 0.30 & 2.2 & $\mathrm{R}$ & patchy blue \\
\hline 101823.00 & 412741.7 & NGC3184 & $1.1(2.1)$ & 0.51 & 0.29 & 0.06 & 3.8 & $\mathrm{~S}$ & \\
\hline 102506.98 & 170947.2 & NGC3239 & $1.4(\mathrm{c} 2 \mathrm{f})$ & 0.66 & 0.34 & 0.14 & 13.9 & $\mathrm{~S}$ & \\
\hline 102508.20 & 170948.3 & NGC3239 & $1.9(\mathrm{c} 2 \mathrm{f})$ & 0.55 & 0.36 & 0.06 & 8.1 & $\mathrm{~S}$ & \\
\hline 112018.31 & 125900.3 & NGC3627 & $1.2(\mathrm{c} 2 \mathrm{f})$ & 0.98 & 0.50 & 0.27 & 13.7 & $\mathrm{~S}$ & \\
\hline 112020.89 & 125846.1 & NGC3627 & $4.1(\mathrm{c} 2 \mathrm{f})$ & 1.77 & 0.53 & 0.32 & 1.4 & $\mathrm{R}$ & \\
\hline 112607.33 & 433406.3 & NGC3675 & $1.7(\mathrm{c} 2 \mathrm{f})$ & 1.73 & 0.71 & 0.44 & 3.0 & $\mathrm{~N}$ & patchy blue \\
\hline 115237.36 & 022807.1 & UGC6850 & $1.8(\mathrm{c} 2 \mathrm{f})$ & 0.41 & -0.06 & -0.34 & 13.0 & $\mathrm{~S}$ & \\
\hline 120922.18 & 295559.7 & NGC4136 & $2.1(1.7)$ & 0.35 & 0.26 & -0.07 & 4.5 & $\mathrm{~S}$ & \\
\hline 121033.76 & 302358.0 & NGC4150 & $3.9(\mathrm{c} 2 \mathrm{f})$ & 1.61 & 0.75 & 0.39 & 17.7 & $\mathrm{~N}$ & bulge \\
\hline 121510.91 & 203912.4 & NGC4204 & $1.6(\mathrm{c} 2 \mathrm{f})$ & -0.06 & 0.33 & -0.44 & 1.8 & $\mathrm{R}$ & isolated H II? \\
\hline 122817.83 & 440633.9 & NGC4449 & $0.6(0.8)$ & 0.17 & 0.25 & -0.19 & 63.7 & $\mathrm{~S}$ & \\
\hline 123029.55 & 413927.6 & NGC4490 & $1.1(1.0)$ & 0.81 & 0.75 & -0.30 & 15.5 & $\mathrm{~N}(\mathrm{~S})$ & patchy blue, H II? \\
\hline 123030.82 & 413911.5 & NGC4490 & $2.7(2.6)$ & 0.54 & 0.30 & 0.18 & 18.7 & $\mathrm{~N}$ & patchy blue \\
\hline 123032.27 & 413918.1 & NGC4490 & $2.0(1.7)$ & 0.74 & 0.40 & 0.10 & 14.1 & $\mathrm{~S}$ & \\
\hline 123036.32 & 413837.8 & NGC4490 & $2.1(1.8)$ & 1.12 & 0.37 & 0.22 & 45.3 & $\mathrm{~N}$ & bulge \\
\hline 123043.26 & 413818.4 & NGC4490 & $3.8(3.1)$ & 0.68 & 0.26 & 0.10 & 14.8 & $\mathrm{~S}$ & \\
\hline 123551.71 & 275604.1 & NGC4559 & $22.9(9.4)$ & 0.28 & -0.08 & -0.06 & 1.8 & $\mathrm{R}$ & patchy blue \\
\hline 123557.79 & 275807.4 & NGC4559 & $2.8(1.8)$ & 0.76 & 0.32 & 0.22 & 21.4 & $\mathrm{~S}(\mathrm{~N})$ & \\
\hline 123558.56 & 275741.9 & NGC4559 & $15.0(11.1)$ & 1.11 & 0.59 & 0.31 & 15.5 & $\mathrm{~N}$ & dust lane \\
\hline 123608.90 & 191955.9 & NGC4561 & $3.0(\mathrm{c} 2 \mathrm{f})$ & 0.57 & 0.35 & -0.04 & 1.9 & $\mathrm{R}$ & patchy blue \\
\hline 124129.14 & 410757.7 & NGC4618 & $1.7(\mathrm{c} 2 \mathrm{f})$ & 0.54 & 0.23 & 0.09 & 4.6 & $\mathrm{~S}$ & \\
\hline 124152.72 & 411631.7 & NGC4625 & $1.3(\mathrm{c} 2 \mathrm{f})$ & 1.00 & 0.51 & 0.21 & 20.5 & $\mathrm{~S}(\mathrm{~N})$ & \\
\hline 125025.70 & 253129.8 & NGC4725 & $1.6(1.8)$ & 0.50 & 0.60 & -0.10 & 9.1 & $\mathrm{~S}$ & H II? \\
\hline 125026.37 & 253319.4 & NGC4725 & $2.1(2.0)$ & -0.21 & 0.41 & 1.11 & 0.7 & $\mathrm{R}$ & \\
\hline 125027.39 & 253026.5 & NGC4725 & $1.0(0.8)$ & 2.04 & 0.88 & 0.48 & 6.4 & $\mathrm{~N}$ & bulge \\
\hline 125036.88 & 253028.4 & NGC4725 & $1.8(1.6)$ & 1.23 & 0.79 & 0.52 & 0.9 & $\mathrm{R}$ & \\
\hline 125512.31 & 000751.9 & UGC8041 & $2.4(\mathrm{c} 2 \mathrm{f})$ & 0.24 & 0.08 & 0.05 & 6.0 & $\mathrm{~S}$ & \\
\hline 131519.54 & 420302.3 & NGC5055 & $6.0(13.5)$ & 0.73 & 0.40 & 0.30 & 2.2 & $\mathrm{R}$ & \\
\hline 131539.33 & 420153.4 & NGC5055 & $0.4(0.6)$ & 1.17 & 0.69 & 0.34 & 8.2 & $\mathrm{~S}(\mathrm{~N})$ & \\
\hline 131602.27 & 420153.6 & NGC5055 & $1.5(1.3)$ & 1.12 & 0.69 & 0.18 & 6.5 & $\mathrm{~S}$ & \\
\hline 132938.61 & 582505.6 & NGC5204 & $4.7(1.2)$ & 0.69 & 0.23 & 0.14 & 26.8 & $\mathrm{~S}(\mathrm{~N})$ & \\
\hline 140332.39 & 542102.9 & NGC5457 & $3.4(2.0)$ & 0.96 & 0.34 & 0.23 & 8.6 & $\mathrm{~S}(\mathrm{~N})$ & \\
\hline 140414.29 & 542603.8 & NGC5457 & $1.7(1.2)$ & 0.52 & 0.08 & 0.74 & 1.2 & $\mathrm{R}$ & \\
\hline 140459.74 & 533808.9 & NGC5474 & $5.1(\mathrm{c} 2 \mathrm{f})$ & 0.92 & 0.30 & 0.25 & 8.4 & $\mathrm{~N}$ & patchy blue \\
\hline 141939.39 & 564137.8 & NGC5585 & $2.8(\mathrm{c} 2 \mathrm{f})$ & -0.31 & 0.78 & -0.60 & 1.1 & $\mathrm{R}$ & isolated H II? \\
\hline 141952.14 & 564417.3 & NGC5585 & $1.0(\mathrm{c} 2 \mathrm{f})$ & 1.08 & 0.38 & 0.28 & 3.7 & $\mathrm{~N}$ & patchy blue \\
\hline
\end{tabular}

a Observed luminosity estimated from count rates, values from spectral fits in parentheses. "c2f" denotes too few counts for spectral fitting.

${ }^{\mathrm{b}}$ Average colors within $100 \times 100 \mathrm{pc}^{2}$ region.

${ }^{\mathrm{c}}$ SDSS detection significance above sky level in $\sigma$.

${ }^{\mathrm{d}}$ Group designation $R$ for rejected, $S$ for star-forming, and $N$ for non-star-forming according to the empirical criteria, 3.1. values in parentheses according to the Fisher criterion, 3.2 when different. 
TABLE 3

SDSS COLOR Distributions

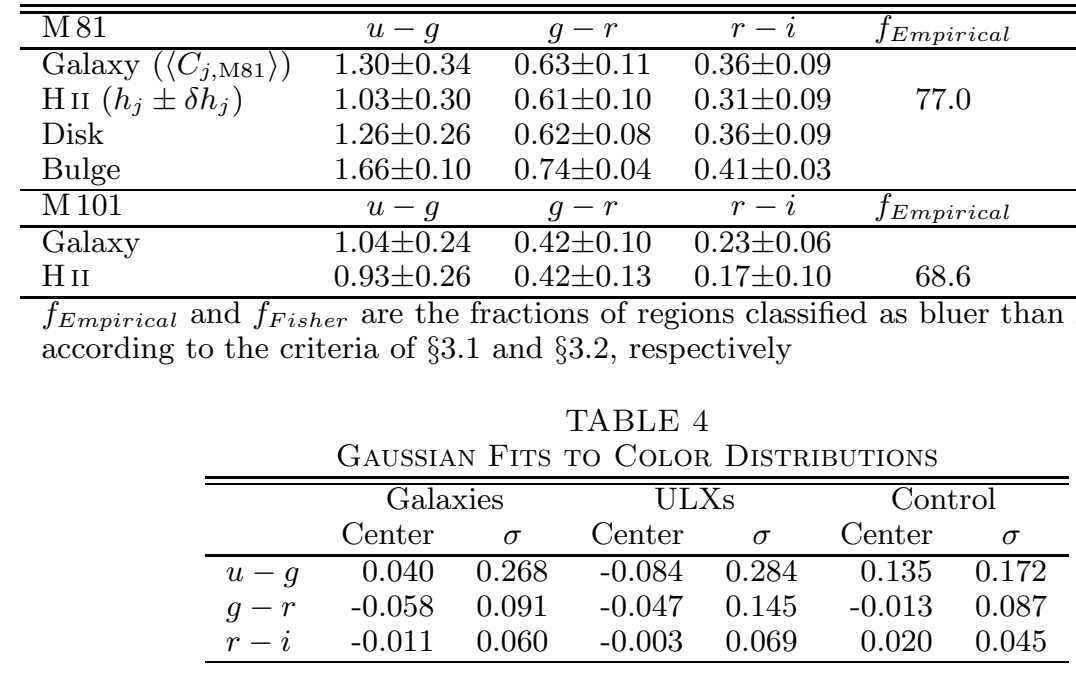



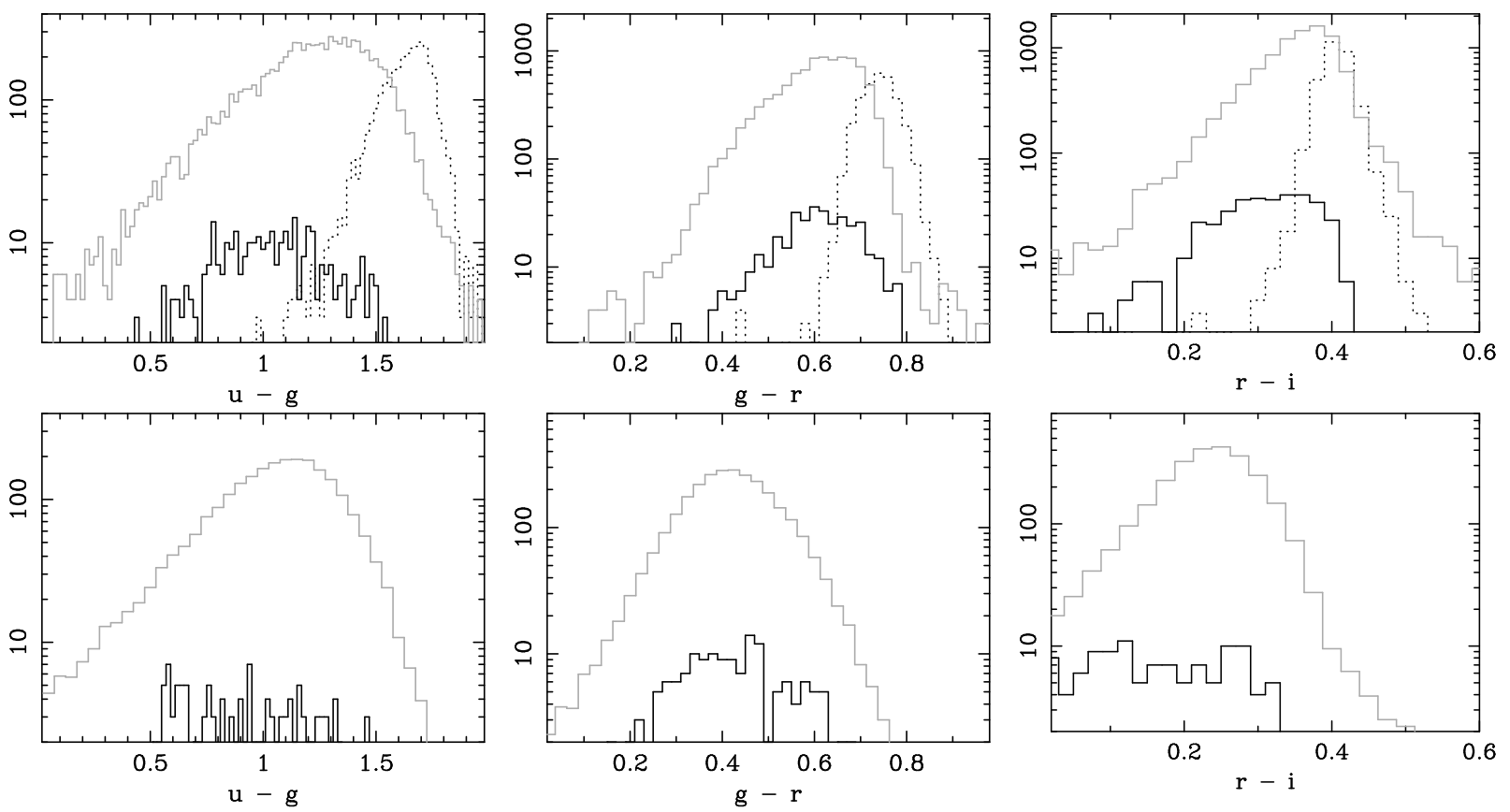

FIG. 1. - Color distributions in the bulge, disk, and H II regions of M 81 (top) and the disk and H II regions of M 101 (bottom). Left-to-right: $u-g, g-r$, and $r-i$ colors of all $100 \times 100 \mathrm{pc}^{2}$ regions in the color map; Heavy line: H II regions, light line: disk, dotted line: bulge.
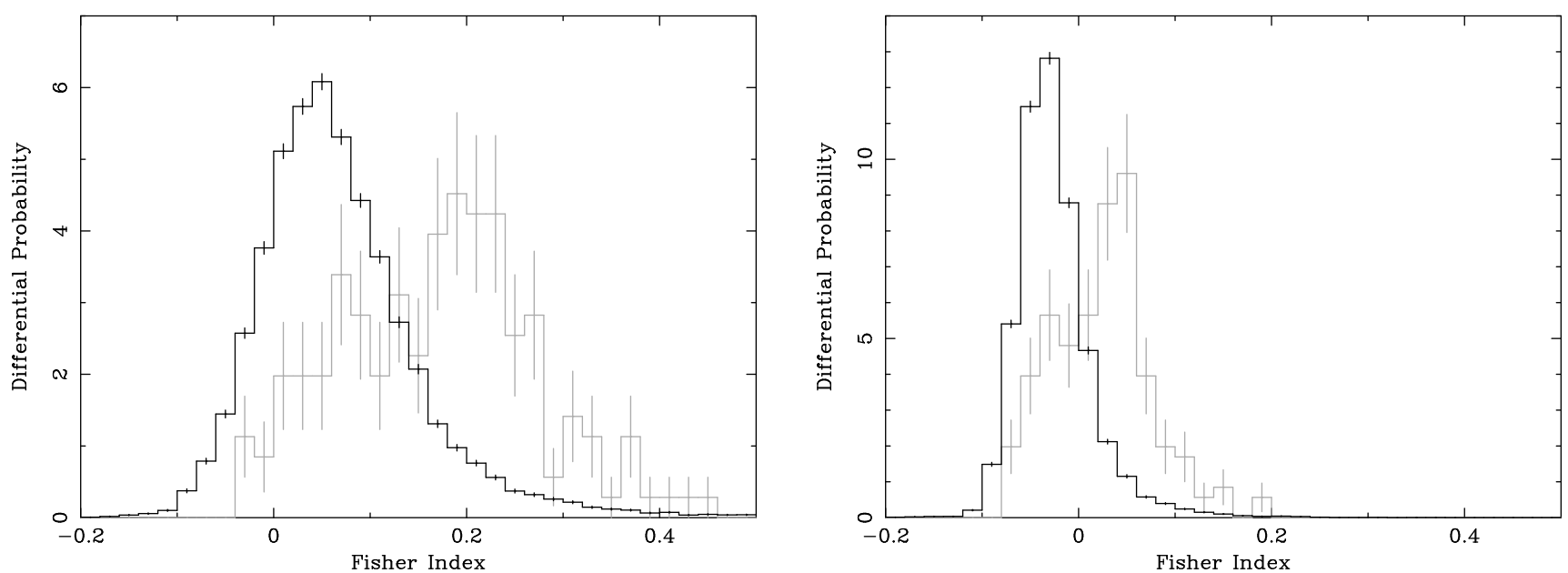

FIG. 2.- Left: Image of the central field of M 81 with all regions classified as star-forming according to the Fisher linear discriminant $(F>0$, Equation 2) highlighted. This result can be be compared to the empirical method displayed in the rightmost panel of Figure 1 Middle: Distribution of all $100 \times 100 \mathrm{pc}^{2}$ regions of M 101 in the Fisher index (heavy line) using the M 81 H II regions as the training set (Equation 2). The light line traces the histogram of the known H II regions in M 101. The area under each histogram is normalized to unity. Right: Same as middle panel but with the Fisher index trained using the H II regions of M 101. Note the fiducial $F=0$ separator applies here but that $F \approx 0.1$ would be more appropriate for the result shown in the middle panel. See text for further discussion. 


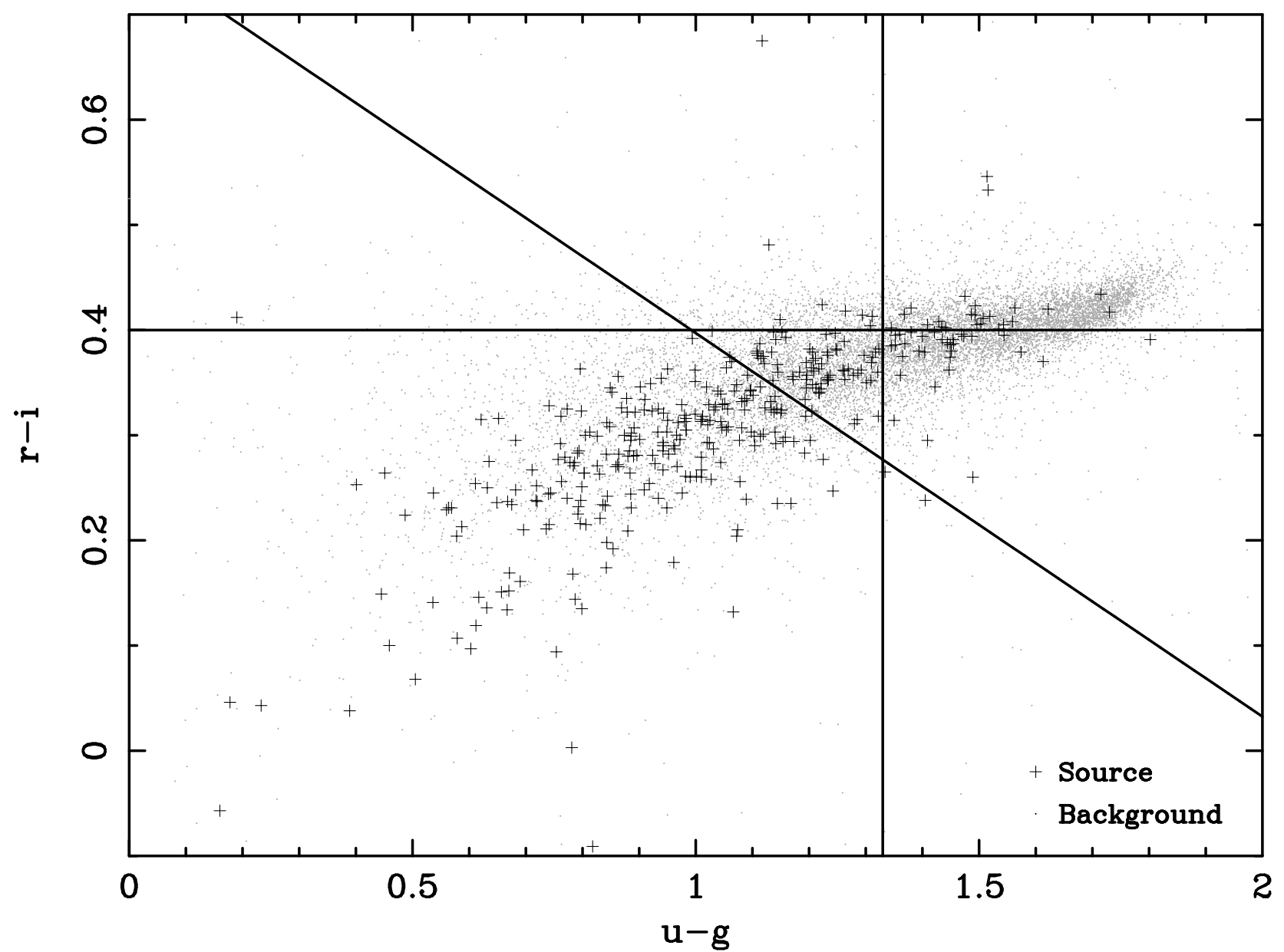

FIG. 3.- Color-color diagram of the $100 \times 100 \mathrm{pc}^{2}$ regions in M 81 . Known $\mathrm{H}$ in regions (Petit et al., 1988) are indicated by crosses. The horizontal and vertical lines are the empirical delineations (Equation 1 ) and the diagonal line is the Fisher index, $F \equiv 0$ (Equation 2 ) separating blue star-forming regions below and to the left of these lines and red bulge and disk regions above and to the right.
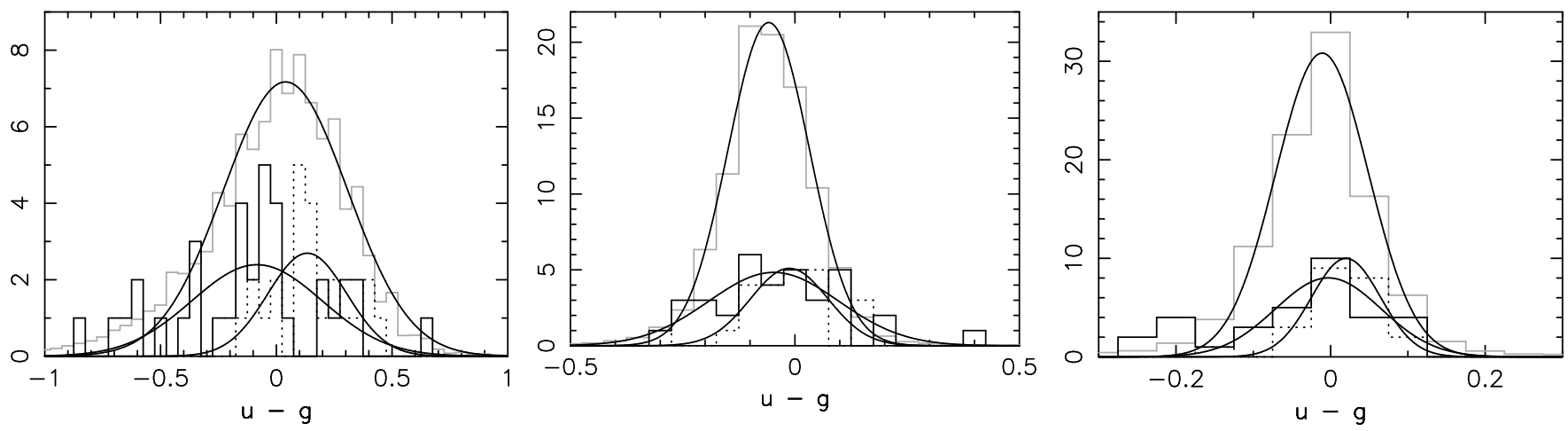

FIG. 4.- Left-to-right: $u-g, g-r$, and $r-i$ color distributions for all resolution elements in all galaxies (light lines), all ULX regions (heavy lines), and all control regions (dotted lines) with significance $\geq 3 \sigma$ above the local sky background. Smooth curves are Gaussian function fits to the histograms. 